Int. J. Electrochem. Sci., 13 (2018) 9359 - 9373

International Journal of

ELECTROCHEMICAL

SCIENCE

www.electrochemsci.org

\title{
Synthesis of a Conductive Pt-Bi/CAG composite and its application for Methyl Orange Decolorization
}

\author{
Lin $\mathrm{Li}^{1}$, Jin-lei Xiong ${ }^{1}$, Luo-chun Wang ${ }^{1, *}$, Tian Xin-mei ${ }^{1}$, Zi-yang Lou ${ }^{2}$, Shou-qiang Huang ${ }^{2}$, \\ Zhen Zhou $^{l}$ \\ ${ }^{1}$ College of Environmental and Chemical Engineering, Shanghai University of Electric Power, \\ Shanghai 200090, China, \\ ${ }^{2}$ School of Environmental Science and Engineering, Shanghai Jiao Tong University, Shanghai \\ 200240, China \\ *E-mail: wangluochun@ shiep.edu.cn
}

doi: $10.20964 / 2018.10 .11$

Received: 4 June 2018 / Accepted: 16 July 2018 / Published: 1 September 2018

To improve the electrochemical properties of electrodes and reduce their cost, a special multi-layer matrix was synthesized as an electrode material, which was composed of cement, active carbon (AC), and graphite with Pt-Bi co-deposition (Pt-Bi/CAG). Pt-Bi particles were found to be homogeneously distributed on the surface of the GAC electrode according to the SEM, EDS and XRD patterns, and the Pt-Bi/CAG-G5 electrode, which was made up of 5\% graphite, $20 \%$ AC powder, $25 \%$ cement and $50 \%$ water, showed the best electrochemical performance in terms of specific capacitance, corrosion resistance and catalytic performance. The specific capacitance of the Pt-Bi/CAG-G5 electrode was 1.103 times larger than that of the Pt-Bi/CAG-G7.5 electrode, and the corrosion resistance was slightly lower than that of the Pt-Bi/CAG-G0 electrode. The current density was appreciably larger than those of all other electrodes, and it was 1.5 times larger than that of the Pt-Bi/CAG-G7.5 electrode. Next, the electrode was optimized based on methyl orange (MO) decoloration experiments, and approximately $76.62 \%$ of the decoloration rate was obtained by the Pt-Bi/CAG-G5 electrode, with a corresponding TOC removal rate of $54.80 \%$ in 180 min under a square wave potential $\left(\mathrm{E}_{\mathrm{U}} 1.5 \mathrm{~V}, \mathrm{E}_{\mathrm{L}}-1.5 \mathrm{~V}\right)$. This composite electrode provides a cost-efficient application using electrochemical technologies (ETs) for the removal of refractory compounds.

Keywords: cement/AC/graphite electrode; electrochemical properties; multi-layer-matrix; costefficiency

FULL TEXT 
(C) 2018 The Authors. Published by ESG (www.electrochemsci.org). This article is an open access article distributed under the terms and conditions of the Creative Commons Attribution license (http://creativecommons.org/licenses/by/4.0/). 